\title{
A Case Study on Wildfire Propagation Modelling Using Evolving Rule Based Fuzzy Cognitive Maps
}

\author{
Joao P. Carvalho and Pedro Gregório \\ INESC-ID / Instituto Superior Técnico, Universidade de Lisboa \\ R. Alves Redol, 9, 1000-029, Lisboa, Portugal \\ joao.carvalho@inesc-id.pt, g.pedro@mail.telepac.pt
}

\begin{abstract}
Fuzzy Cognitive Maps (FCM) and other Dynamic Cognitive Maps (DCM) were originally developed as mechanisms to simulate the evolution of complex qualitative dynamic systems. In this paper we present a case study where we show that Evolving DCM (specifically Evolving Rule Based Fuzzy Cognitive Maps, Ev-RBFCM) can also be used as a valid qualitative option to model a complex physical dynamic system, the propagation of a wildfire.
\end{abstract}

Keywords: Fuzzy Cognitive Maps; RuleBased Fuzzy Cognitive Maps; Dynamic Cognitive Maps; Evolving temporal modelling; Wildfire Propagation.

\section{Introduction}

In 1986 Bart Kosko introduced Fuzzy Cognitive Maps (FCM) [3][4][5] as a tool to model the dynamics of qualitative systems such as Social, Economic or Political systems. Such systems are composed of a number of dynamic qualitative concepts interrelated in complex ways, usually including feedback links that propagate influences in complicated chains, that make reaching conclusions by simple structural analysis an utterly impossible task.

FCM are a combination of Neural Networks and Fuzzy Logic that allow us to predict the change of the concepts represented in Causal Maps. The graphical illustration of FCM is a signed directed graph with feedback, consisting of $\mathrm{n}$ nodes and weighted interconnections. Nodes of the graph stand for the concepts that are used to describe the behavior of the system and they are connected by signed and weighted arcs representing the causal relationships that exist among concepts. FCM representation can usually be reduced to a $\mathrm{n} \times \mathrm{n}$ matrix where each position contains a weight representing the causal relation between the concept indicated in the respective row and the concept indicated in the respective column. FCM have been extensively studied and applied. Extensive details about FCM representation, inference and the associated semantics can be found in [20].

Since the introduction of FCM, hundreds of works have applied FCM or FCM variants to the most diverse fields. However, FCM have limitations in what concerns the modelling and simulation of the dynamics of CM [11][14][20]. Several FCM extensions or variations have been proposed throughout the years to deal with those limitations. Some of them stay very true to the basic mechanisms of FCM, while others present a rather different approach. Usually each approach has its own designation (e.g.: DCN - Dynamical Cognitive Networks [28], E-FCM - Extended Fuzzy Cognitive Maps [21], RB-FCM - Rule Based Fuzzy Cognitive Maps [14], etc.); here we use the term Dynamic Cognitive Maps (DCM) to roughly cover such approaches.

Rule Based Fuzzy Cognitive Maps (RB-FCM), are a particular case of DCM since they are fuzzy rule based systems, as opposed to other DCM, which are closely related to Neural Networks. RB-FCM allow a representation of the dynamics of complex real-world qualitative systems with feedback, and the simulation of events and their influence in the system [14]. They can be represented as fuzzy directed graphs with feedback, and are composed of fuzzy nodes (Concepts), and fuzzy links (Relations). RB-FCM are true cognitive maps since are not limited to the representation of causal relations. Concepts are fuzzy variables described by linguistic terms, and Relations are defined with fuzzy rule bases. RB-FCM are essentially iterative fuzzy rule based systems where fuzzy mechanisms to deal with feedback were added, timing mechanisms were introduced, new ways to deal with uncertainty propagation were defined, and several kinds of concepts (Levels, Variations) and relations (Causal, Inference, Alternatives, Probabilistic, Opposition, Conjunction, etc.) were proposed in order to cope with the complexity and diversity of the dynamic qualitative systems being modeled [11][12][14][16]. Among new contributions brought by RB-FCM, there is a new fuzzy operation, the Fuzzy Carry Accumulation, which is essential to model the mechanisms of qualitative causal relations (FCR - Fuzzy Causal Relations) while maintaining the simplicity and versatility of FCM [20]. In order to better model the dynamics of the modeled systems, two main classes of Concepts were proposed: Levels, 
that represent the absolute values of system entities (e.g., LInflation is Good); and Variations, that represent the change in value of a system entity in a given amount of time (e.g., VInflation increased very much).

One of the things that most DCM have in common is the fact that their cognitive configuration is usually static, i.e., once the cognitive map is modeled, neither its concept structure, nor the relations that define the system behavior, change through time. This fact hinders DCM capabilities to model the temporal behavior of complex real-world systems, especially when the time span to be modeled and simulated is very large. Acampora and Loio [7] explicitly addressed the need for mechanisms that would allow modelling such behavior with the introduction of Timed Automata FCM (TA-FCM) in 2011.

However, the notion of cognitive map Meta-states, introduced in 2006 [16][17], can implicitly model such mechanisms on a qualitative and simpler way. Such principles can be applied to implement Evolving Dynamic Cognitive Maps (Ev-DCM). The use of the term "Evolving" is not related with the mechanisms of what is commonly known as "Evolutionary systems". It refers to the fact that the structure of the map and its composing elements evolve throught time: concepts can be deleted, new concepts might be introduced, new relations can appear, and existing relations can change, etc. I.e., the cognitive map's structure is no longer static.

In this paper we argue that Ev-DCM can also be used to implement qualitative models of complex physical systems (which are a priori pure quantitative systems). In this paper we use a wildfire occurred in 2001 in Portugal as case study to show that Ev-DCM can be successfully used in such way.

\section{Evolving Dynamic Cognitive Maps}

Evolving Dynamic Cognitive Maps (Ev-DCM) were originally introduced in [15], but have only recently been formalized [18]. They were developed in order to address several temporal related problems that arise when modelling qualitative dynamic systems: 1 - The need to model different temporal scales in the same $\mathrm{CM} ; 2$ - The natural evolution of the cognitive configuration of the modelled system; 3 - Drastic changes in the structure of the modelled system (normally due to external/uncontrolable factors).

Ev-DCM are implemented via a Finite State Machine Transducer (FSM) mechanism where each state is designated as Meta-state.

An Ev-DCM consists of several meta-states organized as a deterministic FSM transducer where each metastate is a DCM. An Ev-DCM is a septuple

$$
\left(\Sigma, \Gamma, M S, m s_{0}, \delta, \omega, b_{\text {time }}\right),
$$

where:
- $\quad \Sigma$ is the input alphabet. It defines the inputs for the transition conditions (events).

- $\quad \Gamma$ is the output alphabet. It defines the possible outputs for the EV-DCM and consists of all possible states of all the DCM contained in the EV-DCM.

- $\quad M S$ is a finite and non-empty set of meta-states. Each meta-state is a DCM.

- $m s_{0}$ is the initial meta-state and an element of $M S$, i.e., $m s_{0} \in M S$.

- $\delta$ is a possibly time dependent closed state transition function, $\delta: M S \times \Sigma \rightarrow M S$. It defines the conditions for the transitions and the next meta-state according to the set of events.

- $\omega$ is the output function, and it is a function of the current meta-state and the input alphabet, i.e., $\omega: M S \times \Sigma \rightarrow \Gamma$. It corresponds to the simulation of the active DCM.

- $\quad b_{\text {time }}$ is the current iteration [12]. Only one metastate can be active in each $b_{\text {time }}$ instant.

The DCM associated with each meta-state is simulated only and only if the corresponding meta-state is active. Such DCM is referred to as "current DCM".

An Ev-DCM is therefore composed of a finite number of states (here called meta-states), possible transitions, a set of inputs used to define transition conditions (events), and actions (outputs). The possible states of all DCM that compose the Ev-DCM correspond to the set of outputs $(\Gamma)$ of the Ev-DCM. The output function $\omega$ is the simulation of the current DCM until the occurrence of an event that generates a transition to a new meta-state.

The FSM is used to represent the evolution of an EvDCM. Since each meta-state can be represented by totally distinct DCMs, this mechanism allows a huge versatility in the type of changes that can be modeled through meta-state transitions. More details can be found in [18].

When the DCM is a RB-FCM, then the model is called an Ev-RBFCM.

\section{$3 \quad$ Wildfire modelling}

Wildfire propagation is a complex spatial and temporal dynamic process that depends on a large number of uncertain and imprecise factors such as wind, slope, type of vegetation (also known as fuel), temperature, humidity, etc. Many approaches to wildfire propagation modelling have been proposed and studied, from pure "crisp" mathematical models (usually based on the Rothermel equations), to cellular automata and models based on machine learning and computational intelligence techniques. Despite varying degrees of success, no model can claim to be an ideal solution to the problem due to several reasons: 
- Pure crisp models demand very complex and precise data, and cannot deal with the variety of real world terrain and the uncertainty of fire propagation factors. Additionaly, most dynamic models do not integrate well with GIS [25] due to fundamental incompatibilities in conceptual representation of space and time [2][23];

- Cellular automata models have improved through time (especially with the use of Voronoi region based cells [8][27]) but are still limited in what concerns modelling fire behavior in each individual cell and, therefore, fire propagation [9][22][26];

- Individual computational intelligence techniques have an obvious potential but, up to date, most solutions have limited applicability and results [10][22].

\section{Wildfire Ev-DCM}

In this work we model wildfire propagation based on:

1) A Voronoi based fuzzy Cellular Automata (previously introduced and described in [16][17]), used to build a spatial model of the terrain where the fire propagates;

2) Ev-RBFCM, used to model the temporal dynamic behavior of the fire within a cell.

Each Voronoi cell is computed in order to represent a reasonably homogeneous propagation terrain. Homogeneity depends on factors such as slope, fuel, etc. Each of these cells has a given burning potential that is dependent not only on the cell characteristics (slope, fuel, area, etc.), but also on current weather conditions (temperature, wind speed and direction, etc.), and the state of the neighbouring cells. The behavior of a cell is modelled by an Ev-RBFCM containing the four Meta-States represented in Fig. 1.

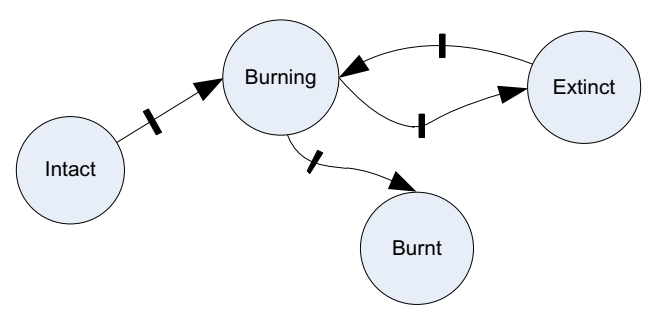

Figure 1: FSM of the Voronoi Based Wildfire Ev-RBFCM.

Meta-states Intact and Extinct share the same basic RB-FCM (Fig.5), but the relations between the concepts have some have different rules, since it is easier to ignite a recently extinct fire, than to start a new one. Transition from these two states to state Burning happens when the concept "Ignition Factor" reaches a certain target value. This value is dependent on the state of the neighbor cells and the atmospheric conditions. The RB-FCM includes concepts such as Wind, Temperature, Convection, Radiation, Combustion Intensity, Distance, Propagation Rate, etc., and several types of inference, causal and Variation-to-Level relations (FIR, FCR, LV [14]). FCR- are "negative causal relations", i.e., causal relations where an increase in the origin concept leads to a decrease in the destination concept. A detailed description of each concept (including linguistic membership functions) and relations (including the fuzzy inference rules), can be found in [24] - the document is in Portuguese, but all the rules, membership functions and technical details concerning the model are graphically depicted and easily understandable. Note that this RB-FCM is a very particular case of a cognitive map since it does not include any cycle.

Meta-state Burning (Fig. 6) is a RB-FCM that models how fast a cell burns considering several complex and uncertain factors (temperature, moisture, fuel, etc.). Fire might become extinct either by lack of fuel, or due to external factors (firemen action, climatic changes, etc.). Details can be found in [24]. While in the Burning state, this cell affects its neighbours (i.e., some of its concpets are used as an input for the RBFCM of the neighbour cells). The transition from Burning to Extinct occurs if concept Fire Intensity becomes zero. If the cell totally burns, cell fuel becomes zero and the model transits to meta-state Burnt.

Meta-state Burnt, is a non-dynamic final state that simply indicates that the whole cell has burned. Hence it is not modeled with an RB-FCM.

Is should be noted that important factors such as, e.g., fuel computations based on the type of vegetation, are based on fuzzy adaptations of crisp equations with a strong theorical background.

This Ev-RBFCM has produced very interesting results allowing a quick and rather accurate modelling based on very uncertain online obtainable data. The model also operates almost automatically, and no decisions have to be taken by experts except for starting the model and getting the results. Online real time data can be used by the model during runtime without any particular difficulty. For example, firemen can send information they gather locally regarding the state of a particular area (local wind direction, burnt areas, etc.). Such data is introduced automatically in the model replacing the corresponding simulated values, and the model continues the simulation taking into account the new data. The Ev-RBFCM was successfully integrated on the Computational Intelligence Distributed System for Forest Fire Combat Aid prototype (STOPFire).

\section{Case study: the 2001 Trémoa wildfire}

In order to test and validate the final model on a real world scenario, we used the data from a wildfire in the region of Trémoa, district of Coimbra, Portugal, [6]. 
The wildfire ignited in two locations (both sides of a small road) at 15:55 on September 5th, 2001, and was automatically detected by the Bosque System surveillance cameras [1]. The fire was considered extinct at 22:30 (due to firefighting action). Fig.2 shows a topographic map of the area, the ignition points, and the final burnt area (in purple).

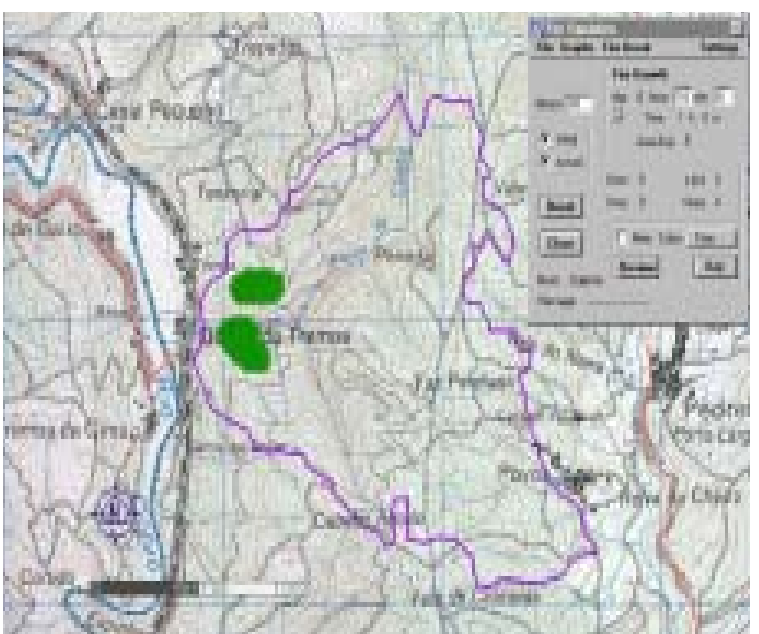

Figure 2: Forest fire in Trémoa, 2001/09/05. Green shows the fire spread from the two ignition areas shortly after the fire detection (the diameter of the longer area is approximately $200 \mathrm{~m}$ ). The purple line shows the burnt area after the fire extinction, approximately 7 hours after ignition (extracted from [6]).

Terrain features are publicly available (including fuel characterization [6]) and were used to compute the Voronoi cells represented in Fig.3. Meteorological data from a nearby station (S. Pedro Dias) is also publicly available, and was registed hourly (Air temperature, relative humidity, wind velocity and direction).

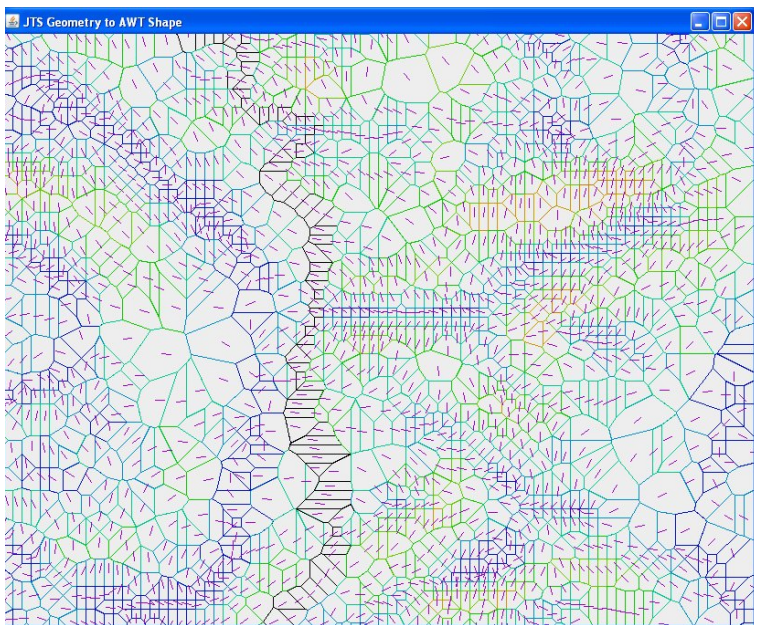

Figure 3: The Trémoa wildfire Voronoi cells. The colored straight lines in the center of the cells represent slope direction, bleck indicates a road, the remaining colours represent slope degree (from blue $=$ flat, to red $=$ very_steep)
The model was fed with the data, and simmulated the predicted wildfire propagation. Figure 4. Shows the Ev-RBFCM fire propagation prediction 4 hours after ignition (each shade of orange represents 1 hour in the simmulation). The scale is identical to the scale of Figure 2. Firemen were only able to start combating the fire efficiently at the 3 hours mark due to the terrain topography (the exception was an initial attack on the south flank).

Wnem comparing Figures 2. and 3., it is possible to conclude that the simulation (prediction) is very consistent with the observed fire spread until the 3 hour mark. The prediction shows a slight spread to the south that is not visible in the actual fire but can be explained by the initial firemen action. The final (7 hour) shape of the burnt area (Figure 2., purple line) is not totally consistent with the Ev-RBFCM fire prediction development because of the firemen action and their efforts in protecting the village situated to the right/center of the image (which are factors that are obviously not considered in the prediciton model).

Considering that all terrain data and geographical conditions are generic and can be automatically obtained online, the prediction of the developed system is very accurate. If available at the time of the real fire, the simmulation could have been used to predict the very violent initial stages and prevent the loss of two fire combat vehicles.

We should note that no locally obtained real time data was given to the model during the simulation to tune the initial predictions, and that the expert knowledge containd in the model rulebases was not tuned to this particular scenario.

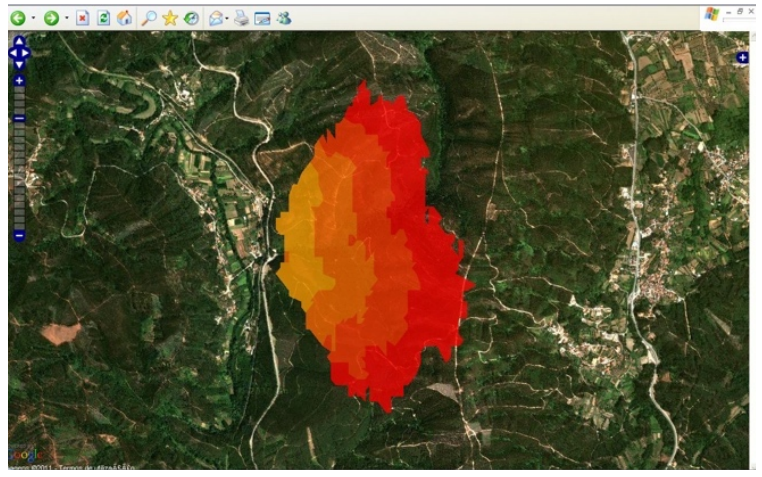

Figure 4: STOPFire fire propagation prediction after 4 hours, given initial wind and moisture conditions, and online available terrain and fuel data. Each shade of orange represents 1 hour of prediction.

\section{Conclusions}

This paper presents a real world case study of a qualitative wildfire propagation model based on an Ev-RBFCM, and shows that, even though DCM were not originally developed to model the dynamics of physical systems, they can be a valid and efficient tool for such purpose. 


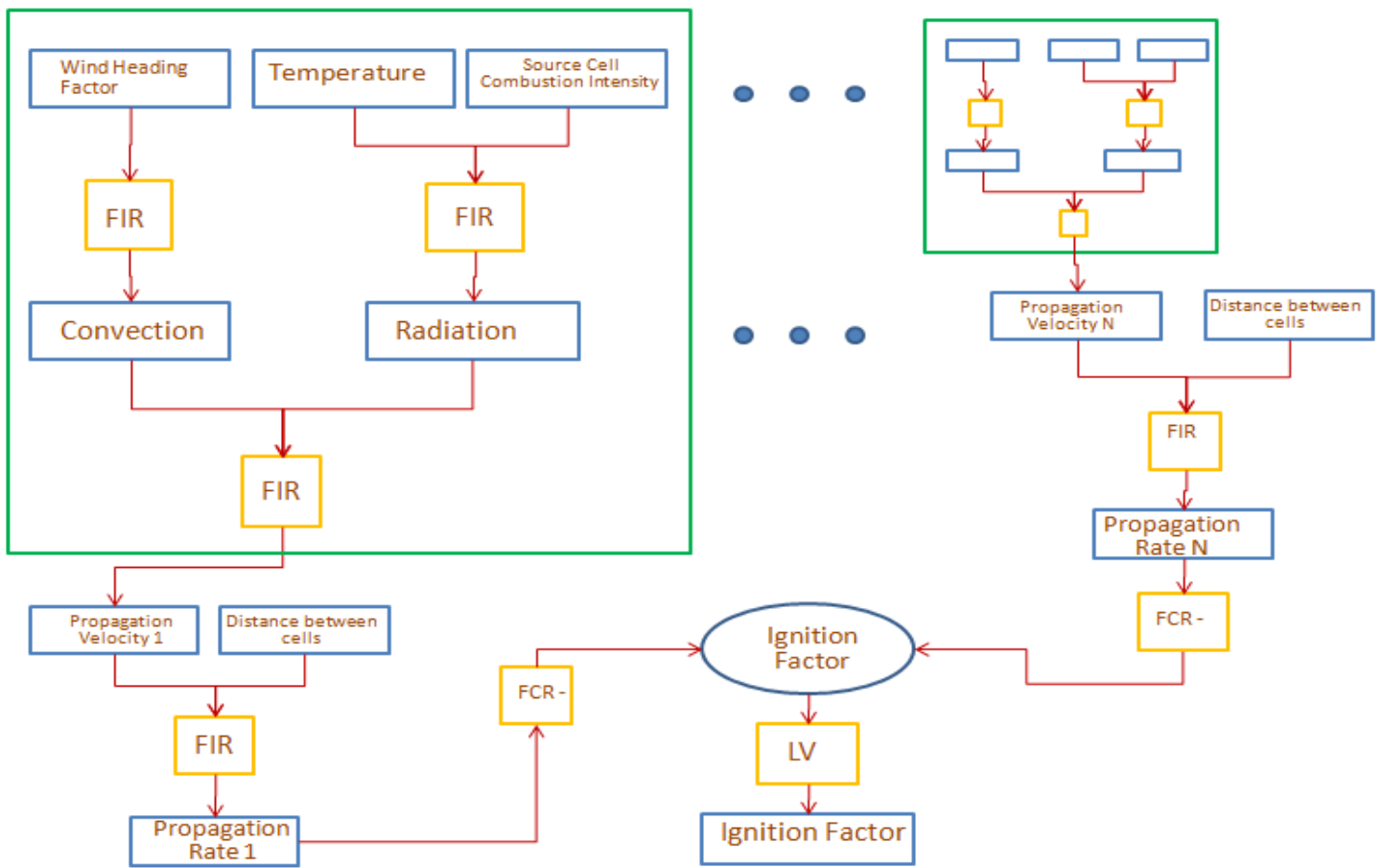

Figure 5: Meta States "Intact" and "Extinct" in Voronoi Based Wildfire Ev-RBFCM

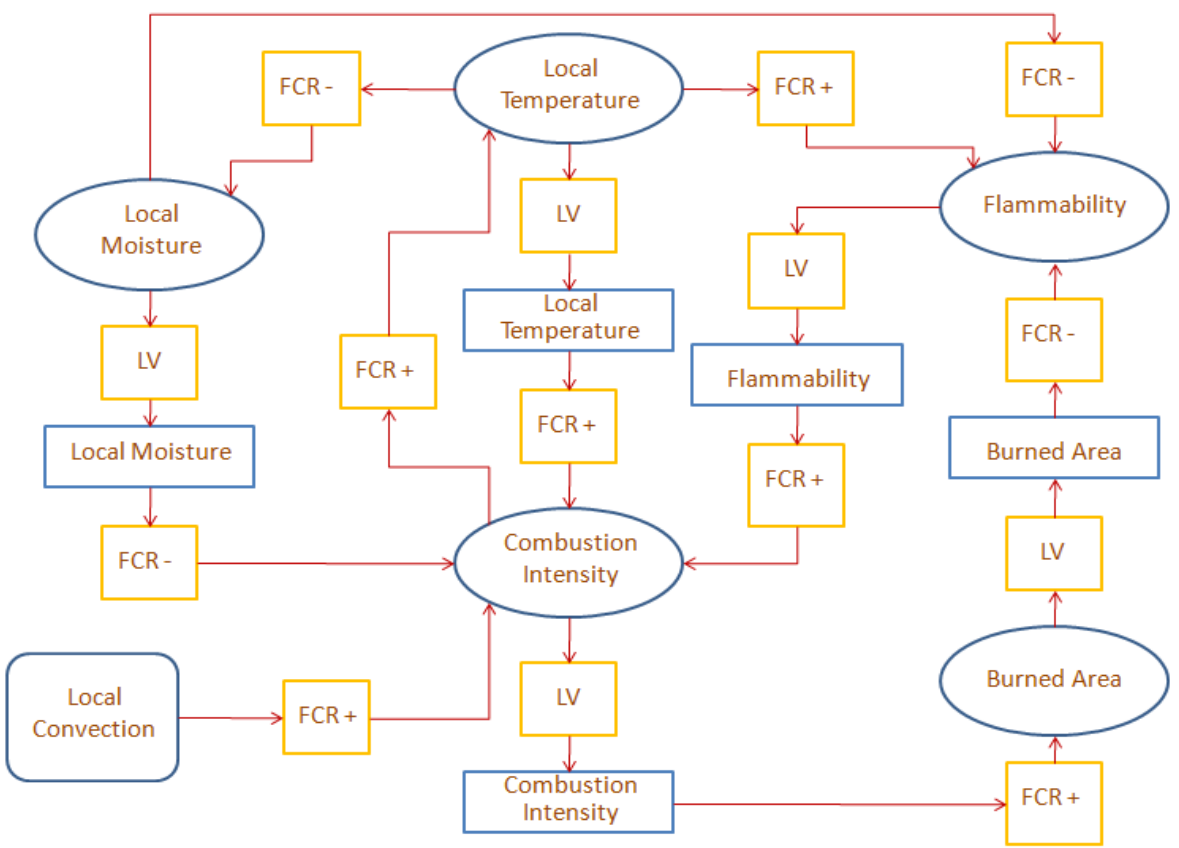

Figure 6: Meta-state "Burning" in Voronoi Based Wildfire Ev-RBFCM. 


\section{Acknowledgements}

Work supported by national funds through Fundação para a Ciência e a Tecnologia (FCT) under reference UID/CEC/50021/2019, grant SFRH/BSAB/136312/ 2018 and projects LISBOA-01-0145-FEDER-031474 and PCIF/SSI/0010/2018

\section{References}

[1] A. Gandia, A. Criado, M. Rallo, El Sistema Bosque, Alta Tecnologia en Defensa del Medio Ambiente. DYNA. 1994;6:34-38.

[2] A.M.G. Lopes, M.G. Cruz, D.X. Viegas, FireStation - An Integrated Software System for the Numerical Simulation of Fire Spread on Complex Topography, Environmental Modelling \& Software 17(2002) 269-285, 2002

[3] B. Kosko, Fuzzy Cognitive Maps, International Journal of Man-Machine Studies, vol.24 (1), pp.65-75, 1986

[4] B. Kosko, Fuzzy Thinking, Hyperion, 1993

[5] B. Kosko, Neural Networks and Fuzzy Systems: A Dynamical Systems Approach to Machine Intelligence, Prentice-Hall International Editions, 1992

[6] D.X. Viegas, L. Ribeiro, P. Palheiro, Trémoa Forest Fire in Central Portugal: A case study of fire behaviour prediction, in Forest Fire Research \& Wildland Fire Safety: Proceedings of IV International Conference on Forest Fire Research / 2002 Wildland Fire Safety Summit, Coimbra, Portugal, 2002.

[7] G. Acampora, V. Loia, On the Temporal Granularity in Fuzzy Cognitive Maps. IEEE Trans. on Fuzzy Syst. 9 (6) pp 1040-1057, 2011

[8] H. Shiyuan, L. Deren, Vector Cellular Automata Based Geographical Entity, Geoinformatics 2004, Proc. 12th Int. Conf. on Geoinformatics, ài Geospatial Information Research: Bridging the Pacific and Atlantic University of Govle, Sweden, 2004

[9] I. Karafyllidis, A. Thanailakis, A Model for Predicting Forest Fire Spreading Using Cellular Automata, Ecological Modelling 99, 87-97, 1999

[10] J.A. Olivas, Forest Fire Prediction and Management using Soft Computin, INDIN'03, Bannf, Canada, 2003.

[11] J.P. Carvalho, J.A. Tomé, Fuzzy Mechanisms for Qualitative Causal Relations, Views on Fuzzy Sets and Systems from Different Perspectives. Philosophy and Logic, Criticisms and Applications. (Studies in Fuzziness and Soft
Computing), Chapter 19, Edited by. Rudolf Seising, Springer, 2009

[12] J.P. Carvalho, J.A. Tomé, Rule Based Fuzzy Cognitive Maps - Expressing Time in Qualitative System Dynamics, Proceedings of the 2001 FUZZ-IEEE conference, Melbourne, Australia, 2001

[13] J.P. Carvalho, J.A. Tomé, Rule Based Fuzzy Cognitive Maps - Fuzzy Causal Relations, Computational Intelligence for Modelling, Control and Automation: Evolutionary Computation \& Fuzzy Logic for Intelligent Control, Knowledge Acquisition \& Information Retrieval, edited by M. Mohammadian, IOS Press, 1999

[14] J.P. Carvalho, J.A. Tomé, Rule Based Fuzzy Cognitive Maps - Qualitative Systems Dynamics, Proceedings of the 19th International Conference of the North American Fuzzy Information Processing Society, NAFIPS2000, Atlanta, pp. 407-411, 2000

[15] J.P. Carvalho, L. Wise, A. Murta, M. Mesquita, Issues on Dynamic Cognitive Map Modelling of Purse-seine Fishing Skippers Behavior, Proc. of the WCCI2008 - 2008 IEEE World Congress on Computational Intelligence, Hong-Kong, pp. 1503-1510, 2008

[16] J.P. Carvalho, M. Carola, J.A. Tome, Forest Fire Modelling using Rule-Based Fuzzy Cognitive Maps and Voronoi Based Cellular Automata, Proceedings of the 25th International Conference of the North American Fuzzy Information Processing Society, NAFIPS 2006, Montreal, Canada, 2006

[17] J.P. Carvalho, M. Carola, J.A. Tomé, Using Rule Based Fuzzy Cognitive Maps to Model Dynamic Cell Behaviour in Voronoi Based Cellular Automata, Proc. of the WCCI2006 - 2006 IEEE World Congress on Computational Intelligence, pp. 1503-1510, 2006

[18] J.P. Carvalho, On the Implementation of Evolving Dynamic Cognitive Maps, Proceedings of the IFSA - NAFIPS 2019, 2019 IFSA World Congress and NAFIPS Annual Conference, 2019 (accepted)

[19] J.P. Carvalho, On the Semantics and the Use of Fuzzy Cognitive Maps in Social Sciences, Proc. of the WCCI2010 - 2010 IEEE World Congress on Computational Intelligence, Barcelona, pp. 2456-2461, 2010

[20] J.P. Carvalho, On the Semantics and the Use of Fuzzy Cognitive Maps and Dynamic Cognitive Maps in Social Sciences. Fuzzy Sets and Systems, Vol 214-1, pp 6-19, 2013

[21] M. Hagiwara, Extended Fuzzy Cognitive Maps, in proceedings of IEEE International Conference on Fuzzy Systems, pp. 795-801, 1992 
[22] M. Mraz, N. Zimic, J. Virant,Predicting Wind Driven Wild Land Fire Shape Using Fuzzy Logic in Cellular Automata, Proc. Of the ISAI/IFIS, 1996

[23] N. Hazelton, F. Leahy, I. Williamson, Integrating Dynamic Modelling and Geographic Information Systems", Journal of URISA, 4(2), 47-58, 1992

[24] P. Gregório, J.P. Carvalho, Stop Fire: An Evolving Rule-Based Fuzzy Cognitive Map and Voronoi Based Cellular Automata WildFire Propagation Simulator - Technical Annex Part A, https://www.inescid.pt/ficheiros/publicacoes/14531.pdf, 2019

[25] P.A. Burrough, R.A., McDonnell, Principles of Geographical Information Systems, Oxford University Press, Oxford, 1998
[26] S.G. Berjak, J.W. Hearne, An Improved Cellular Automaton Model for Simulating Fire in a Spatially Heterogeneous Savanna System, Ecological Modelling, 148 (2002) 133-151, 2002

[27] W. Shi, M.Y. Cheung Pang, Development of Voronoi-Based Cellular Automata-An Integrated Dynamic Model for Geographical Information Systems, Int. Journal of Geographical Information Science, vol. 14, no. 5, 455 474 , 2000

[28] Y. Miao, Z. Liu, C. Siew, C. Miao, Dynamical Cognitive Network - an Extension of Fuzzy Cognitive Map, IEEE Transactions on Fuzzy Systems, vol. 9, no. 5, pp. 760-770, 2001 